Marquette University

e-Publications@Marquette

Physics Faculty Research and Publications

Physics, Department of

$11-1-2016$

Magnetization Reversal in Ferromagnetic Spirals via Domain Wall Motion

Ryan D. Schumm

Marquette University

Andrew Kunz

Marquette University, andrew.kunz@marquette.edu

Published version. Applied Physics Letters, Vol. 109, No. 2 (November 2016). DOI. (C) 2016 American Institute of Physics. Used with permission. 


\title{
Magnetization reversal in ferromagnetic spirals via domain wall motion
}

\author{
Ryan D. Schumm and Andrew Kunz \\ Physics Department, Marquette University, Milwaukee, Wisconsin 53233, USA
}

(Received 3 August 2016; accepted 7 November 2016; published online 16 November 2016)

\begin{abstract}
Domain wall dynamics have been investigated in a variety of ferromagnetic nanostructures for potential applications in logic, sensing, and recording. We present a combination of analytic and simulated results describing the reliable field driven motion of a domain wall through the arms of a ferromagnetic spiral nanowire. The spiral geometry is capable of taking advantage of the benefits of both straight and circular wires. Measurements of the in-plane components of the spirals' magnetization can be used to determine the angular location of the domain wall, impacting the magnetoresistive applications dependent on the domain wall location. The spirals' magnetization components are found to depend on the spiral parameters: the initial radius and spacing between spiral arms, along with the domain wall location. The magnetization is independent of the parameters of the rotating field used to move the domain wall, and therefore the model is valid for current induced domain wall motion as well. The speed of the domain wall is found to depend on the frequency of the rotating driving field, and the domain wall speeds can be reliably varied over several orders of magnitude. We further demonstrate a technique capable of injecting multiple domain walls and show the reliable and unidirectional motion of domain walls through the arms of the spiral. Published by AIP Publishing. [http://dx.doi.org/10.1063/1.4968012]
\end{abstract}

Understanding and controlling the magnetization reversal in magnetic nanostructures is important for a variety of applications in logic, sensing, and recording. ${ }^{1-3}$ In many of these technologies, the magnetization reversal is facilitated by the motion of a domain wall (DW) moving through the structure. In ferromagnetic nanostructures, the shape anisotropy plays an outsized roll in the dynamics of the reversal process and numerous studies have been completed on DW motion in a variety of geometries including stripes, wires, and rings. ${ }^{4-7}$ Reliable DW motion is critical for magnetoresistance (MR) applications where the value of the MR depends on the relative alignment of the magnetization across a layer stack. In wire geometries, the relative alignment between layers can be varied by moving a DW, and by injecting/removing DWs. These processes give rise to changes in the MR values, both continuous and discrete. ${ }^{8-11}$ In this study, we use a combination of analytic modeling and micromagnetic simulation to investigate the magnetization reversal in permalloy nanospiral wires due to the motion of a DW driven by an in-plane rotating magnetic field.

Spiral nanowires present an opportunity to combine the strengths of motion in straight wires and nanorings with avoiding the complications. In nanorings, it is impossible to set up a situation where there is only one DW and as such the magnetic signature is either zero (in the vortex state with no DWs) or dependent on the direction of the applied field (onion state). Conversely, because the spiral is not closed, a single DW can be propagated through the spiral arms and single DW dynamics can be investigated in nearly ring like geometries. In comparison with the straight wires, the coiling of the spiral allows for much longer wires in a shorter areas footprint (a $56 \mu \mathrm{m}$ long six turn spiral fits within a $5 \mu \mathrm{m} \times 5 \mu \mathrm{m}$ square). In long straight wires, it is possible to have a single DW but the speed of the DW is limited in large magnetic fields because of the Walker breakdown. ${ }^{12}$ Additionally, when more than one DW is present in a straight wire, the neighboring DWs move in opposite directions when driven by magnetic fields, but in the spiral every DW moves in the direction of the rotating field with a speed dependent on the frequency of the rotating field. ${ }^{13,14}$

We investigated the Archimedes spirals that have a constant spacing between neighboring spiral arms, as shown in Fig. $1 .^{15}$ The radius, $r$, is given by $r(\theta)=a \theta+b$ for a given angle $\theta$, where $a$ is related to the spacing between the spiral arms and $b$ is the initial radius when $\theta=0$. While not necessary, we investigate spirals with an integer number of turns, $s$, such that the final rotation angle of the wire is $\theta_{f}=2 \pi s$.

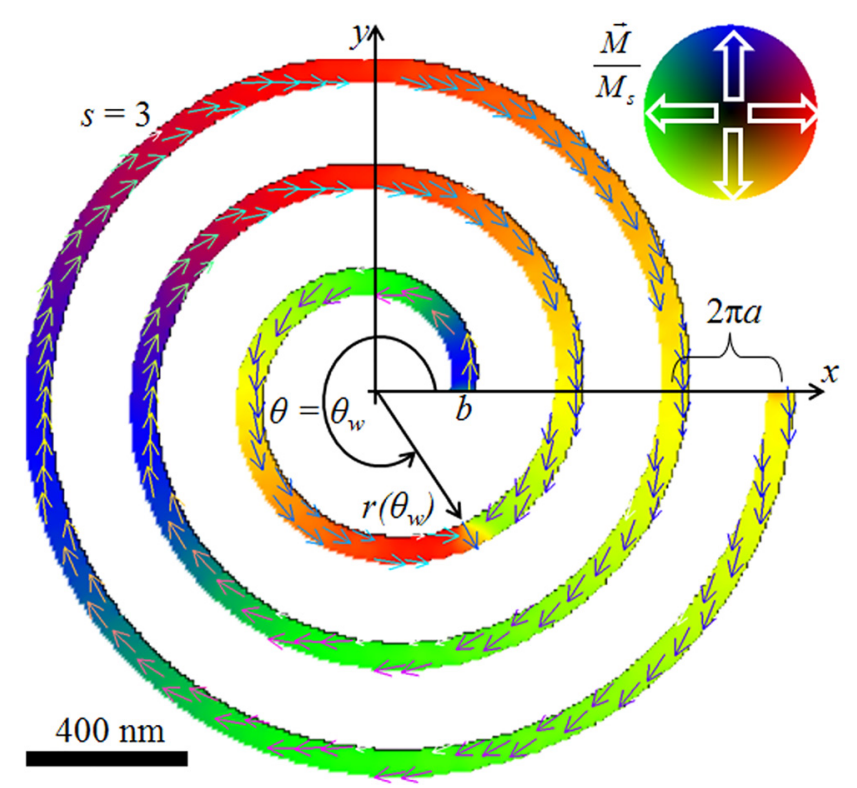

FIG. 1. A representative three turn $(s=3)$ spiral structure for which the reversal of the magnetization is carried out via the motion of a DW driven by an in-plane rotating magnetic field. 
Fig. 1 shows the simulated micromagnetic domain configuration for a three turn $(s=3)$ spiral with a DW located in the interior spiral arm. Permalloy wires with a rectangular crosssection, $100 \mathrm{~nm}$ wide and $5 \mathrm{~nm}$ thick, are modeled to ensure transverse, or Néel, DWs between the in-plane magnetized domains. ${ }^{16}$ The non-magnetic spacing between the arms is $200 \mathrm{~nm}$ and the initial radius is $300 \mathrm{~nm}$. The magnetization reversal is investigated with the standard micromagnetic simulation via integration of the Landau-Lifshitz equation of motion for the three dimensional rotation of the magnetic moments in the wire. The simulation takes into account the long-range magnetostatic and local ferromagnetic exchange interactions, along with the effects of an in-plane rotating magnetic field. The parameters used are appropriate for permalloy with saturation magnetization $M_{s}=8 \times 10^{5} \mathrm{~A} / \mathrm{m}$, exchange constant $A=1.3 \times 10^{-11} \mathrm{~J} / \mathrm{m}$, and damping parameter alpha of 0.01 . The dynamic integration uses a predictorcorrector 4th order algorithm with a time step of less than a picosecond to accurately model the DW dynamics. ${ }^{17}$

In order to investigate the magnetization reversal in the spiral nanowire, a single DW must be located in the spiral. This is initially accomplished by applying a saturating magnetic field along the $y$-axis of the spiral. ${ }^{15}$ The saturating field creates a pair of transverse DWs in each arm of the wire and the subsequent application of an in-plane clockwise rotating field sweeps the DWs through the spiral arms until a single DW remains. Starting from this configuration, the rotating fields of varying strength and frequency are applied to move the DW counterclockwise through the spiral until it leaves the outer arm. The DW location is tracked along with the $x$ - and $y$-components of the magnetization of the spiral as a function of time. Fig. 2(a) shows the angular position of the DW as a function of time as it is driven by a $15 \mathrm{mT}$ field rotating with a frequency of $100 \mathrm{MHz}$. The slope of the line is equal to the frequency at which the field is rotated demonstrating that the domain wall tracks the direction of the magnetic field; the speed of the domain wall depends on the frequency and location as $v=\omega r$, not the strength of the applied field. Figs. 2(b)-2(e) show the snapshots of the magnetic domain configuration of the spiral. The DW moves uniformly through the wire in time following the direction of the applied field as it rotates. The simulated $y$-component of the spirals' magnetization, a quantity that is experimentally accessible, is also shown in Fig. 2(a) as a function of time. ${ }^{15}$ We note that at $t=25 \mathrm{~ns}$ the amplitude of the signal decreases as the DW leaves the spiral. This continued oscillation is due to the continued rotation of the applied magnetic field. The segments of the wire perpendicular to the applied field continue to oscillate as they experience the greatest torque. Field amplitudes between $3.0 \mathrm{mT}$ and $15 \mathrm{mT}$ have been investigated, and the amplitude of this oscillation increases with increasing field strength; the results shown here are for a $15 \mathrm{mT}$ field that has the most uniform motion. The reduced magnetization, defined here to be the magnetization of the wire in zero applied field, shown in Fig. 2(a) is calculated by subtracting the oscillation amplitude from the total simulated amplitude. Expressions for the reduced magnetization components can be calculated with the knowledge of spiral geometry, as shown below.
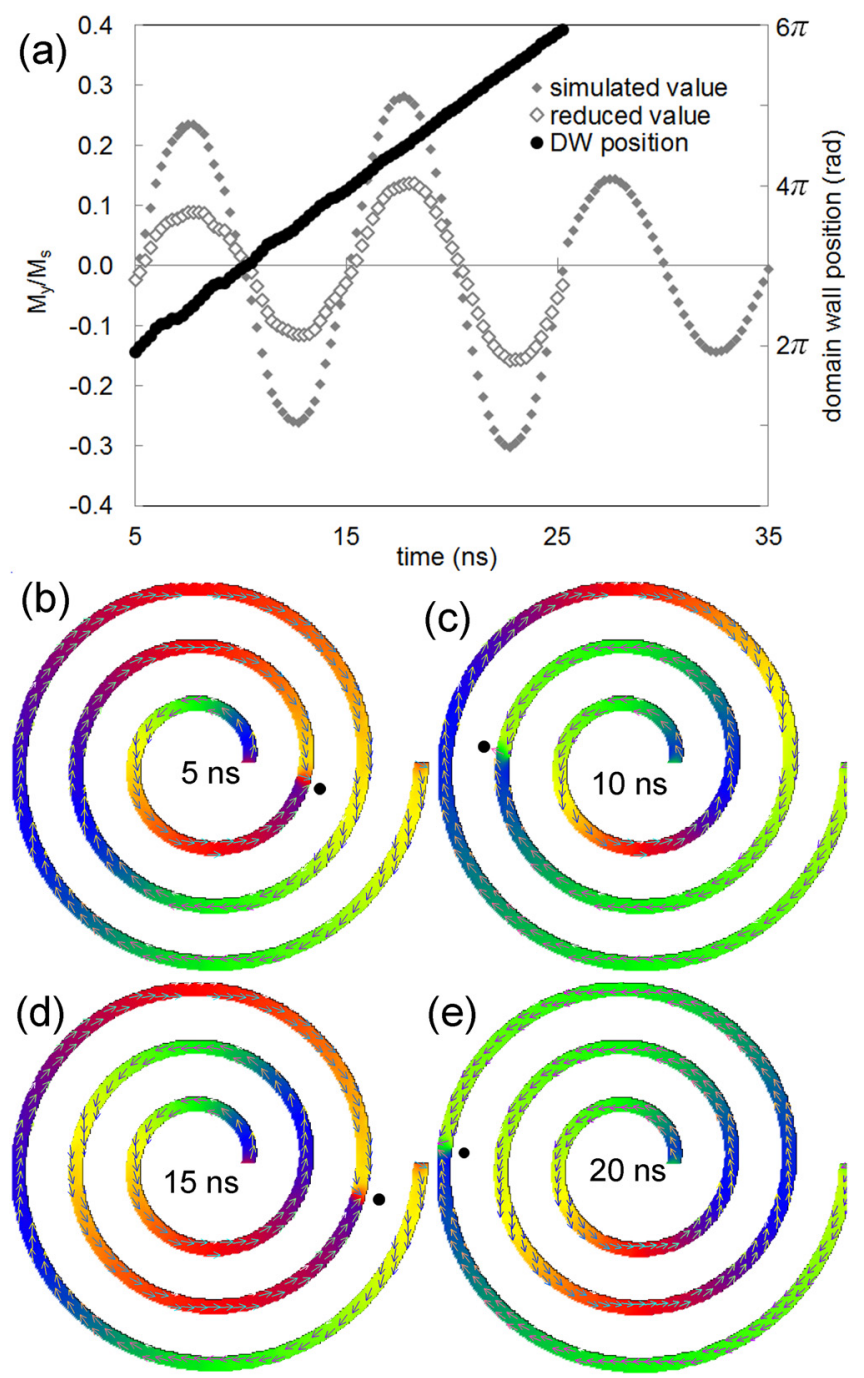

FIG. 2. (a) A DW moves uniformly as a $15 \mathrm{mT}$ driving field is rotated in the plane of the spiral. At $t=25 \mathrm{~ns}$, the DW leaves the spiral. The simulated signal from $25 \mathrm{~ns}<t<35 \mathrm{~ns}$ is removed from the simulated values to create the reduced value. (b)-(e) Snapshots of the domain configuration in the spiral. Dots are located at the DW location to guide the eye.

In practice, it would be useful to know the location of the DW within the spiral. Experimentally measuring the $x$ and $y$-components of the magnetization is sufficient to determine the location. Starting in the uniformly magnetized, i.e., single domain state, we use the fact that the magnetic moments align in the plane and parallel to the axis of the spiral rotation at each location. Summing the magnetic moments along each of the in-plane axes, we find a set of expressions for the magnetization of a spiral segment

$$
\begin{gathered}
\frac{M_{x}}{M_{s}}=\frac{1}{L} \int_{0}^{2 \pi s}[a \cos \theta-(a \theta+b) \sin \theta] d \theta \\
=\left.\frac{(a \theta+b) \cos \theta}{L}\right|_{0} ^{2 \pi s}, \quad \text { and } \\
\frac{M_{y}}{M_{s}}=\frac{1}{L} \int_{0}^{2 \pi s}[a \sin \theta-(a \theta+b) \cos \theta] d \theta=\left.\frac{(a \theta+b) \sin \theta}{L}\right|_{0} ^{2 \pi s},
\end{gathered}
$$

where $a, b, \theta$, and $s$ are the spiral parameters previously defined and $L$ is the physical length of the spiral. We further 
reduce (1) to give the value of the magnetization components for a single domain spiral consisting of an integer number of turns

$$
\begin{gathered}
\frac{M_{x}}{M_{s}}=\frac{2 \pi a}{L} s, \quad \text { and } \\
\frac{M_{y}}{M_{s}}=0 .
\end{gathered}
$$

These equations show that the magnetic moments along the $y$-axis are fully compensated when the spiral consists of full turns, but that the horizontal component is non-zero due to the extra length of the spiral along the $x$-axis. When a DW is placed in the spiral and moved through it, the in-plane components of the magnetization change, as shown in Fig. 2(a), with the set of components uniquely defining the DW location.

The magnetization components of the spiral containing a domain wall can be found by starting with the uniformly magnetized case from (2) and reversing the magnetization to the domain wall location $\left(\theta_{w}\right)$. Therefore, in the case of a single DW, the magnetization can be expressed as

$$
\begin{gathered}
\frac{M_{x}}{M_{s}}=\frac{2}{L}\left[\pi a s-\left(a \theta_{w}+b\right) \cos \theta_{w}+b\right], \quad \text { and } \\
\frac{M_{y}}{M_{s}}=-\frac{2}{L}\left(a \theta_{w}+b\right) \sin \theta_{w},
\end{gathered}
$$

which only depend on the DW location and the geometric parameters of the spiral. In Fig. 3, we plot (3) for each turn in the spiral, s1-s3, along with the reduced magnetization determined via micromagnetic simulation as a function of the DW angle showing good agreement. We plot the values within the unit circle to highlight the change in magnitude as the DW spirals outward. As the DW moves through the spiral arms, the magnitude of reduced magnetization in general increases; this is most apparent at the critical angles $(0, \pi / 2$, $\pi, 3 \pi / 2)$. The magnetization increases in each full rotation at these points by a constant value $4 \pi a / L$ that is easy to see from (3).

The simulations show that DW tracks the rotating magnetic field, within in small lag angle, moving in the direction of the rotating field, with an angular speed given by the rotational frequency of the field for all frequencies investigated between $100 \mathrm{kHz}$ and $100 \mathrm{MHz} .^{18-20}$ The tracking is
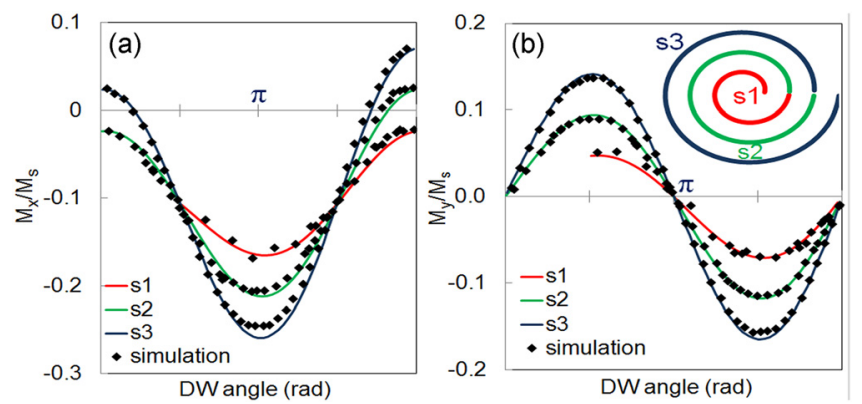

FIG. 3. (a) $x$-and (b) $y$-comparisons of the simulated reduced magnetization showing agreement with the analytic expression as a function of the DW location. In general, the signal grows as the DW spirals from the inner arm (s1) to the outer arm (s3), as defined in the inset. consistent with that found in rings even at slower rotational frequencies. ${ }^{19,20}$ Because the radius of the spiral increases as the DW moves to the outer rings, the tangential speed, $v=\omega r$, of the wall increases with radius, as demonstrated in Fig. 4(a). In the range of frequencies presented, the DW speed varies from about $3 \mathrm{~m} / \mathrm{s}$ to nearly $1000 \mathrm{~m} / \mathrm{s}$ without undergoing Walker breakdown. The tracking behavior allows for expressing the values found in (3) as a function of time, $t$, where $\theta_{w}=2 \pi f t$. These dynamic expressions only depend on the spiral parameters and the rotational frequency such that for every DW location there is a single combination of values for the in-plane components of the magnetization. The independence of the driving mechanism is shown in Fig. 4(b), where the $x$-component of the magnetization is plotted for three different frequencies when the explicit time dependence is removed. The independence on the details of the driving field means that the results are valid for current induced DW motion as well.

Finally note from Fig. 2 that while the $15 \mathrm{mT}$ field is sufficient for moving the DW through the spiral, it is not strong enough to inject DWs into the spiral. This implies that in a stacked spiral geometry it is possible to vary the magnetization of one layer, and therefore the relative alignment in the stack, useful for position (rotation) dependent MR applications. It is possible however to inject a single DW, or multiple DWs, into the spiral using the pad, as shown in Fig. $5 .^{21,22}$ The $300 \mathrm{~nm}$ diameter disk injects DWs with an applied field as low as $5 \mathrm{mT}$. The rotating field smoothly rotates the magnetic moments within the pad and can be used to inject a DW with constant chirality every $\pi$ rotation. ${ }^{21}$ By operating in this manner, each ring can hold two DWs, separated by an angle of $\pi$. Each DW follows the rotation of the magnetic field, moving in the same direction essentially in phase with each other, as shown in Fig. 5. It is also possible to vary the field strength to inject, or not inject, a DW as desired.

In the case of repeated DW injection, the number of DWs and their location can be experimentally determined by measuring the in-plane components of the magnetization. Similar to the earlier discussion, when a DW is injected, the magnetic moments behind it reverse as it moves. With multiple DWs, the magnetization components change more significantly, as shown in Fig. 5(a), as compared to the single wall case. The components can be expressed as

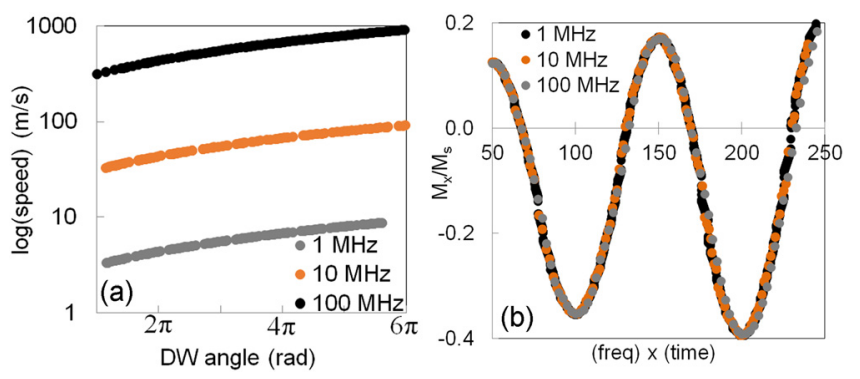

FIG. 4. (a) DW speed as a function of angle for three different frequencies. The DW tracks the $15 \mathrm{mT}$ field and increases in speed as the radius of the spiral increases. (b) The x-component of the magnetization plotted against the dimensionless quantity (frequency times time) - a measure of the DW location showing that the magnetization components are independent of the wall dynamics. 

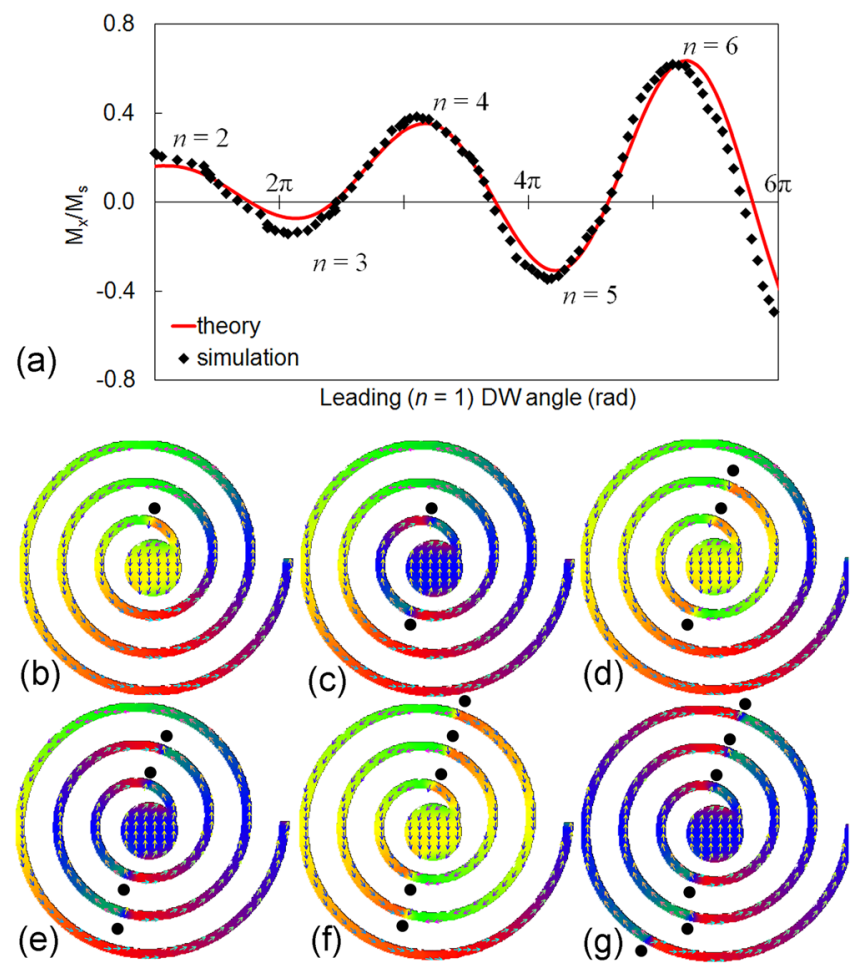

FIG. 5. (a) Comparison of the simulated and calculated horizontal magnetization component of the spiral as six DWs are injected into the spiral at each interval of $\pi$. (b)-(e) Snapshots of the domain configuration as DWs are injected and moved through the spiral by a $15 \mathrm{mT}$ field. Dots are used to guide the eye to the DW locations.

$$
\begin{aligned}
\frac{M_{x}}{M_{s}}= & \frac{2 \pi a}{L} s+\frac{2}{L}\left[\left.\sum_{n=1}^{N-1}(-1)^{n}(a \theta+b) \cos \theta\right|_{\theta_{n}-\pi} ^{\theta_{n}}\right] \\
& +\left.\frac{2}{L}(-1)^{N}(a \theta+b) \cos \theta\right|_{\theta=0} ^{\theta=\theta_{N}}, \text { and } \\
\frac{M_{y}}{M_{s}}= & \frac{2}{L}\left[\left.\sum_{n=1}^{N-1}(-1)^{n}(a \theta+b) \sin \theta\right|_{\theta_{n}-\pi} ^{\theta_{n}}\right] \\
& +\left.\frac{2}{L}(-1)^{N}(a \theta+b) \sin \theta\right|_{\theta=0} ^{\theta_{N}},
\end{aligned}
$$

where $n$ is the integer corresponding to each DW as it is injected, $N$ is the total number of DWs in the spiral and $\theta_{n}$ is the location of the $n$th injected domain wall. In (4), the term containing the sum accounts for the alternating magnetization as a DW injects and moves, with $n=1$ corresponding to the first injected domain wall, $n=2$ the second, etc. The limits represent that a new DW injects when the field rotates through an angle of $\pi$. The last term tracks the contribution of the last, Nth DW injected. The results of the simulated injection of six DWs are plotted along with the calculated values from these expressions in Fig. 5(a), showing a good fit as a function of the leading DW angle (the location of the wall corresponding to $n=1$ ). As the leading DW reaches the end of the spiral, the fit is not good but the simulated images show that the DWs are not all in phase (Fig. 5(g)), showing that the use of the angle $\pi$ between DWs is not as accurate. The non-uniform magnetization of the pad, while accounted for as above, may also impact the agreement.

In conclusion, ferromagnetic nanowires formed into spirals can be reversed by driving a DW through the wire with rotating magnetic fields. The motion of the DW is uniform over a large range of rotational frequencies, and the location of the DW can be determined by measuring the in-plane components of the spirals magnetization. Analytic expressions are in good agreement with the simulated values of the in-plane magnetization components as a function of the DW position and are independent of the details of the driving mechanism, dependent only on the geometric parameters of the spiral. Finally, multiple DWs were injected into the spiral and corresponding equations were found capable of determining the number, and location, of the DWs within the spiral. The spiral geometry presents a useful compromise between straight and ring wire geometries.

This work was supported by the National Science Foundation (NSF DMR - 1309094).

${ }^{1}$ D. A. Allwood, G. Xiong, C. C. Faulkner, D. Atkinson, D. Petit, and R. P. Cowburn, Science 309, 1688 (2005).

${ }^{2}$ L. K. Bogard and D. Atkinson, Appl. Phys. Lett. 94, 042511 (2009).

${ }^{3}$ S. S. P. Parkin, M. Hayashi, and L. Thomas, Science 320, 190 (2008).

${ }^{4}$ O. A. Tretiakov, D. Clarke, G.-W. Chern, Y. B. Bazaliy, and O. Tchernyshyov, Phys. Rev. Lett. 100, 127204 (2008).

${ }^{5}$ G. S. D. Beach, C. Nistor, C. Knutson, M. Tsoi, and J. L. Erskine, Nat. Mater. 4, 741 (2005).

${ }^{6}$ J. Rothman, M. Klaui, C. A. F. Vaz, A. Bleloch, J. A. C. Bland, Z. Cui, and R. Speaks, Phys. Rev. Lett. 86(6), 1098 (2001).

${ }^{7}$ F. J. Castano, C. A. Ross, C. Frandsen, A. Eilez, D. Gil, H. I. Smith, M. Redjdal, and F. B. Humphrey, Phys. Rev. B 67, 184425 (2003).

${ }^{8}$ K. Kondou, N. Ohshima, S. Kasai, Y. Nakatani, and T. Ono, Appl. Phys. Express 1, 061302 (2008).

${ }^{9}$ K. Sawada, T. Uemura, M. Masuda, K. Matsuda, and M. Yamamoto, IEEE Trans. Magn 45(10), 3780 (2009).

${ }^{10}$ M. Fukada, M. Yamanouchi, F. Matsukura, and H. Ono, Appl. Phys. Lett. 91, 052503 (2007).

${ }^{11}$ M. Hayashi, L. Thomas, R. Moriya, C. Rettner, and S. S. P. Parkin, Science 320, 209 (2008).

${ }^{12}$ N. L. Schryer and L. R. Walker, J. Appl. Phys. 45(12), 5406 (1974).

${ }^{13}$ A. Kunz, Appl. Phys. Lett. 94, 132502 (2009).

${ }^{14}$ A. Zhukov, J. M. Blanco, A. Chizik, M. Ipatov, V. Rodionova, and V. Zhukova, J. Appl. Phys. 114, 043910 (2013).

${ }^{15}$ A. Westphalen, A. Remhof, and H. Zabel, J. Appl. Phys. 104, 013906 (2008).

${ }^{16}$ R. D. McMichael and M. J. Donahue, IEEE Trans. Magn. 33(5), 4167 (1997).

${ }^{17}$ M. R. Scheinfien, http://llgmicro.home.mindspring.com for LLG Micromagnetics Simuator v2.63c, 2009.

${ }^{18}$ S. C. Lee, Y. J. Cho, U. H. Pi, J. Y. Bae, J. Heo, S. Seo, J. K. Shin, and T. D. Lee, Jpn. J. Appl. Phys. 49, 023002 (2010).

${ }^{19}$ M. Negoita, T. J. Hayward, and D. A. Allwood, Appl. Phys. Lett. 100, 072405 (2012).

${ }^{20}$ M. Negoita, T. J. Hayward, J. A. Miller, and D. A. Allwood, J. Appl. Phys. 114, 013904 (2013).

${ }^{21}$ A. Kunz and S. C. Reiff, Appl. Phys. Lett. 94, 192504 (2009).

${ }^{22}$ Y. Jang, S. R. Bowden, M. Mascaro, J. Unguris, and C. A. Ross, Appl. Phys. Lett. 100, 062407 (2012). 